\title{
Evaluación del desempeño sísmico de edificaciones compuestas de marcos rígidos de acero en zonas hondureñas de alto peligro sísmico
}

\section{Seismic performance evaluation of steel rigid frame buildings in Honduran high seismic hazard zones}

$\underline{\text { V. Martínez Reyes }}{ }^{(*)}$, C. Nungaray Pérez ${ }^{(* *)}$

RESUMEN

El Código Hondureño de Construcción (CHOC) prescribe una metodología de diseño sísmico de marcos rígidos de acero que genera resultados inciertos y permite diseños de marcos rígidos ordinarios de acero (MRO) en zonas de alto peligro sísmico, comprometiendo su desempeño sísmico requerido. Se evalúa el desempeño sísmico de tres edificios de cinco niveles cada uno en la zona urbana hondureña de mayor peligro sísmico con FEMA 440 y FEMA 356. Dos edificios constan de MRO; uno de ellos con los perfiles más ligeros disponibles, el otro, un diseño frágil permitido por el CHOC. El tercero, con marcos rígidos especiales de acero con los perfiles más ligeros disponibles. Los diseños fueron por el UBC 97 y el AISC 2010. Se verificó, por análisis no lineales, estáticos y dinámicos, que el CHOC puede llevar al diseño de marcos rígidos de acero con desempeño sísmico inaceptable, considerando criterios del UBC 97 y AISC 2010.

Palabras clave: Desempeño sísmico, marcos rígidos de acero, CHOC, análisis.

\section{ABSTRACT}

The Honduran Construction Code (CHOC) prescribes a seismic design methodology for steel moment frames which produces uncertain results. CHOC also allows designs of ordinary steel moment frames (OMF) in regions of high seismic hazard which compromise the required seismic performance of such structures. The seismic performance of three fivestory buildings is evaluated in the Honduran urban area of greatest seismic hazard using FEMA 44O and FEMA 356. Two buildings consist of OMF; one of them with the lightest shapes available, the other, a non-ductile design allowed by the CHOC. The third, with special steel moment frames with the lightest shapes available. The designs were by the UBC 97 and the AISC 201O. It was verified by nonlinear static and dynamic analyzes that the CHOC could eventually lead to the design of steel moment frames with unacceptable seismic performance, in accordance with criteria of UBC 97 and AISC 2010.

Keywords: Seismic performance, steel moment frames, CHOC, analysis.

(*) Facultad de Ingeniería, Universidad Tecnológica Centroamericana (UNITEC), Tegucigalpa (Honduras).

(**) Instituto Tecnológico y de Estudios Superiores de Monterrey, Campus Monterrey, Monterrey (México). Persona de contacto/Corresponding author: victor.martinez@unitec.edu.hn (V. Martínez Reyes). ORCID: https://orcid.org/oooo-0oo2-1059-651X (V. Martínez Reyes); https://orcid.org/oooo-0oo1-7083-4535 (C. Nungaray Pérez)

Cómo citar este artículo/Citation: Martínez Reyes, V.; Nungaray Pérez, C. (2019). Evaluación del desempeño sísmico de edificaciones compuestas de marcos rígidos de acero en zonas hondureñas de alto peligro sísmico. Informes de la Construcción, 71(556): e314. https:// doi.org/10.3989/ic.64333

Copyright: (C) 2019 CSIC. Este es un artículo de acceso abierto distribuido bajo los términos de la licencia de uso y distribución Creative Commons Reconocimiento 4.o Internacional (CC BY 4.0). 


\section{INTRODUCCIÓN}

En años recientes se ha visto la necesidad de contar con códigos de diseño sísmico que generen estructuras sismorresistentes con un desempeño sísmico aceptable, esto es, estructuras que sean capaces de no sufrir ciertos niveles específicos de daño frente a la acción de diferentes niveles de excitación sísmica que podrían afectar la estructura durante su vida útil (1). Típicamente se establecen como objetivos que los edificios de importancia normal se mantengan completamente operacionales ante sismos frecuentes de intensidad baja y que garanticen la seguridad de las personas ante sismos poco frecuentes de intensidad alta (2). Estas afirmaciones han quedado sustentadas luego de revisar las experiencias vividas debido a sismos de magnitud moderada tales como el sismo de Northridge, 1994 (3) o el sismo de Kobe, 1995 (4) en los cuales estructuras diseñadas de acuerdo a códigos actuales de esa época, si bien frente a los eventos sísmicos mencionados cumplieron con un objetivo de desempeño orientado a la seguridad de vidas, sufrieron la pérdida de operación completa lo cual se tradujo en pérdidas económicas que alcanzaron límites inaceptables. Estas circunstancias son indicativas del mal desempeño sísmico de tales estructuras, y evidenciaron el cambio que debía implementarse en el enfoque perseguido por los códigos de diseño sísmico de esa época.

Ante esta situación, el Código Hondureño de Construcción (CHOC) (5), creado por una comisión técnica nombrada por el Colegio de Ingenieros Civiles de Honduras, ofrece un contexto normativo en el cual se visualizan las siguientes debilidades:

Las prescripciones para la determinación de fuerzas sísmicas son tomadas de la publicación Uniform Building Code (UBC 94) (6). Se sabe que los procedimientos de diseño por sismo de dicho código siguen una metodología basada en fuerzas; no obstante, esta metodología arroja un panorama incierto sobre la respuesta sísmica actual de la estructura (7). Adicionalmente a esto y, dentro del ámbito del diseño de estructuras sismorresistentes de acero, el código permite que se diseñen en las zonas hondureñas de mayor peligro sísmico marcos rígidos de acero ordinarios, es decir, sin ningún requerimiento especial de ductilidad. De esta manera el uso del código no garantiza diseños de este tipo de estructuras con desempeño sísmico aceptable. Esta posibilidad de riesgo sísmico contrasta con la necesidad del entorno urbano regional de contar con estructuras sismorresistentes con desempeño sísmico aceptable. Es importante mencionar que el CHOC no contiene provisiones sísmicas para estructuras sismorresistentes de acero, solamente indica el efecto que tienen este tipo de estructuras en la determinación de las fuerzas sísmicas de diseño y señala su uso en determinadas condiciones. También es importante destacar que las normas indicadas por el CHOC (UBC 94) para la determinación de la demanda sísmica sufrieron modificaciones importantes (8), las cuales se reflejan en la publicación Uniform Building Code (UBC 97) (9).

Debido a lo anteriormente mencionado y, ante la falta de estudios que aborden la problemática en cuestión de forma directa, es de importancia particular realizar evaluaciones de desempeño sísmico de estructuras diseñadas de acuerdo a normativas vigentes, con el fin de obtener resultados que evidencien el grado de confiabilidad que la normativa hondureña actual ofrece para generar diseños de estructuras con desempeño sísmico aceptable. En vista de ello, el objetivo general de esta investigación es realizar este tipo de evaluacio- nes. En particular este trabajo se centra en una edificación ordinaria (un edificio de oficinas) cuyo sistema sismorresistente consta de marcos rígidos de acero. Para el diseño se considera la zona urbana hondureña de mayor peligro sísmico indicada por el CHOC, la cual corresponde a la ciudad de Choluteca. La demanda sísmica se determina considerando el UBC 97 y empleando análisis con espectros inelásticos de diseño. Se realizan tres diseños siguiendo los lineamientos establecidos por las publicaciones Specification for Structural Steel Buildings (ANSI/AISC 360-10) (10), Seismic Provisions for Structural Steel Buildings (ANSI/AISC 341-10) (11) y empleando el método Diseño con Factores de Carga y Resistencia (LRFD). En los diseños también se consideran los requisitos de rigidez lateral mínima especificados por el UBC 97. En el primer diseño se emplea marcos rígidos ordinarios con las secciones transversales más ligeras disponibles (Diseño 1). En el segundo se considera un panorama bajo el cual se emplean marcos rígidos ordinarios cuya selección particular de perfiles produce un mecanismo de falla indeseable, a pesar de ser permitido por el CHOC (Diseño 2); y en el tercero se emplean marcos rígidos especiales con las secciones transversales más ligeras disponibles (Diseño 3). Seguidamente se evalúa el desempeño sísmico de todos los diseños realizando análisis no lineal estático (se emplea el método mejorado del espectro de capacidad) de acuerdo a las publicaciones Improvement Of Nonlinear Static Seismic Analysis Procedures (FEMA 440) (12) y Prestandard and Commentary for the Seismic Rehabilitation of Buildings (FEMA 356) (13); y análisis no lineal dinámico de tiempo-historia de acuerdo al FEMA 356. Con base en los resultados, se concluye sobre las carencias reflejadas por el CHOC y su necesidad de actualización.

\section{MODELO COMPUTACIONAL}

El modelo computacional se realizó empleando el programa ETABS 2016. La geometría del modelo se muestra en las Figuras 1, 2 y 3. Las columnas mostradas correspondientes al diseño 2 se comentarán posteriormente.

El sistema estructural constó de marcos de acero con designación ASTM A992. Tal y como se observa en las figuras anteriores, los marcos rígidos se ubicaron dentro de los planos bidimensionales X-Z y Y-Z. El sistema de entrepiso consistió en una sección compuesta de losa de concreto sobre tableros de acero, los cuales fueron soportados por vigas secundarias. La carga muerta aplicada sobre las vigas del sistema de entrepiso fue $322.69 \mathrm{~kg} / \mathrm{m}^{2}$ en los niveles 1 al 4 y $272.69 \mathrm{~kg} / \mathrm{m}^{2}$ sobre el nivel de azotea. Estas cargas incluyen el peso de los siguientes componentes: losa de concreto, tableros de acero, divisiones internas (solamente en niveles $1 \mathrm{al}$ 4), cielo falso, instalaciones y piso. También se aplicó una carga por muros en las vigas perimetrales de los niveles 1 al 4 de $570.85 \mathrm{~kg} / \mathrm{m}$. Las cargas vivas aplicadas sobre los modelos fueron tomadas de acuerdo al CHOC. Sobre los niveles 1 al 4 se aplicó una carga de $250 \mathrm{~kg} /$ $\mathrm{m}^{2}$, la cual corresponde a una ocupación de oficinas. Sobre la azotea se aplicó una carga viva de $100 \mathrm{~kg} / \mathrm{m}^{2}$. En la determinación de la carga sísmica según el UBC 97 se consideró un tipo de fuente sísmica A, una distancia a la fuente sísmica conocida más cercana estimada entre 10 y $15 \mathrm{~km}$, un tipo de perfil de suelo predominante en la zona $S_{c}$, un factor de importancia sísmica de 1.00 y un factor de zona sísmica de 0.40. El espectro elástico de diseño tiene la forma mostrada en la Figura 4.

Las ordenadas del espectro elástico se redujeron para propósitos de diseño considerando un factor de modificación 
de respuesta de 3.5 para los marcos rígidos ordinarios y de 8 para los marcos rígidos especiales. Los marcos resistentes a carga gravitacional se diseñaron de acuerdo al ANSI/ AISC 360-10. Los marcos rígidos se diseñaron de acuerdo al ANSI/AISC 341-10. En los tres diseños realizados se empleó el catálogo de perfiles indicado por la publicación Steel Cons- truction Manual (14). En todos los casos la respuesta de la estructura, sin realizar análisis de segundo orden, permitió que los efectos de este tipo de análisis fueran despreciados. Los análisis lineal y no lineal, y el diseño de los marcos rígidos especiales se realizó considerando una sección de viga reducida (RBS).

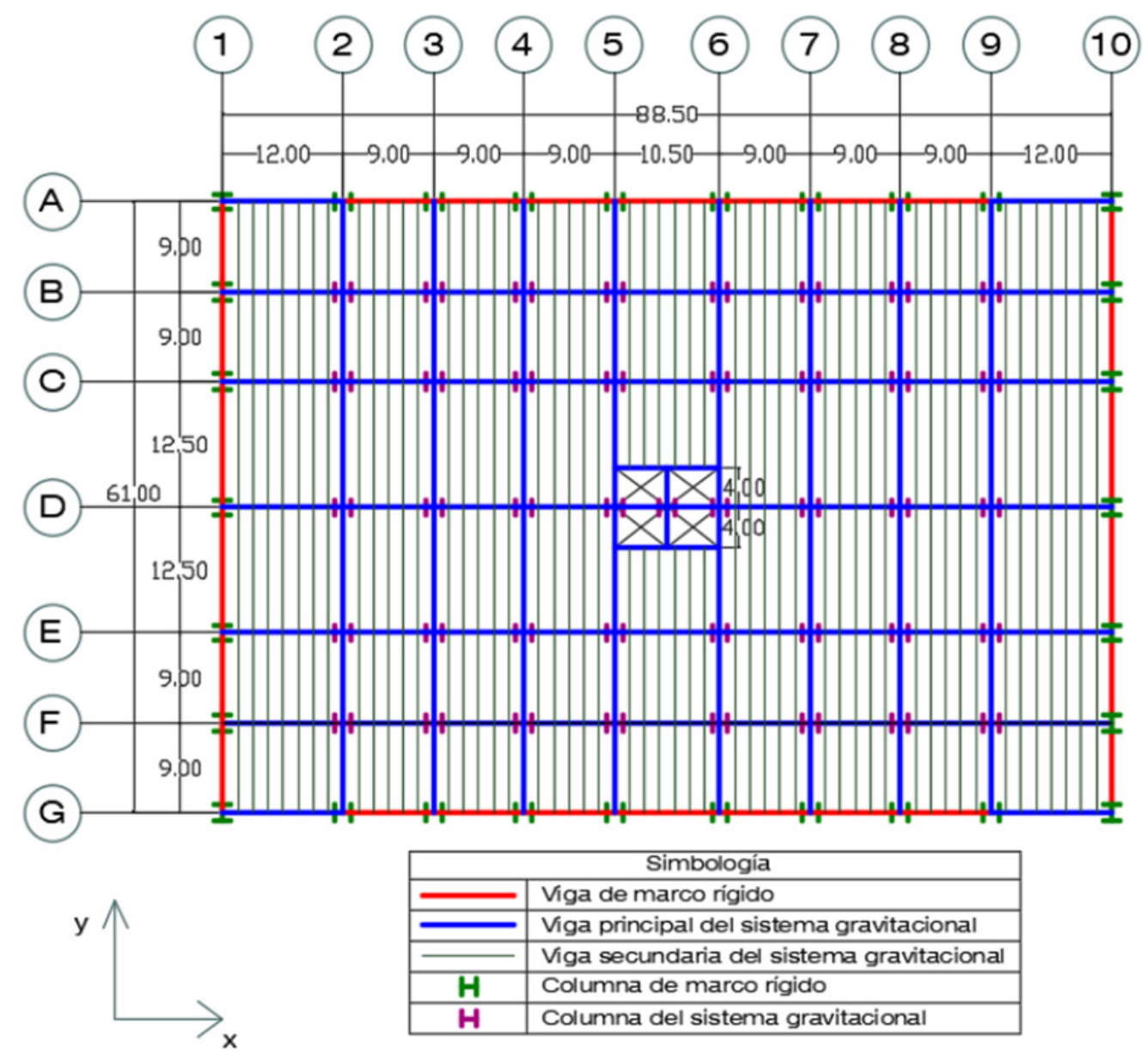

Figura 1. Planta típica (Unidades: Metros).

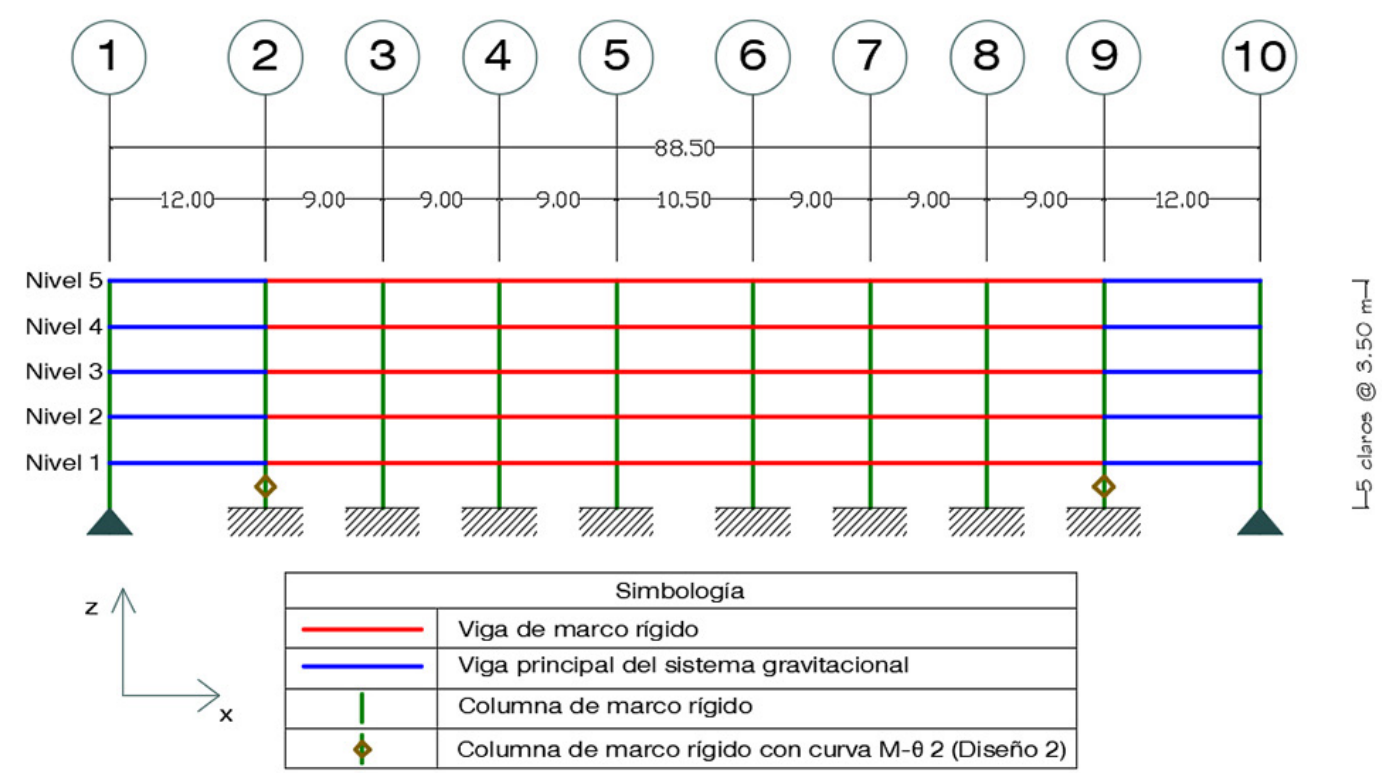

Figura 2. Elevación plano X-Z (Unidades: Metros). 


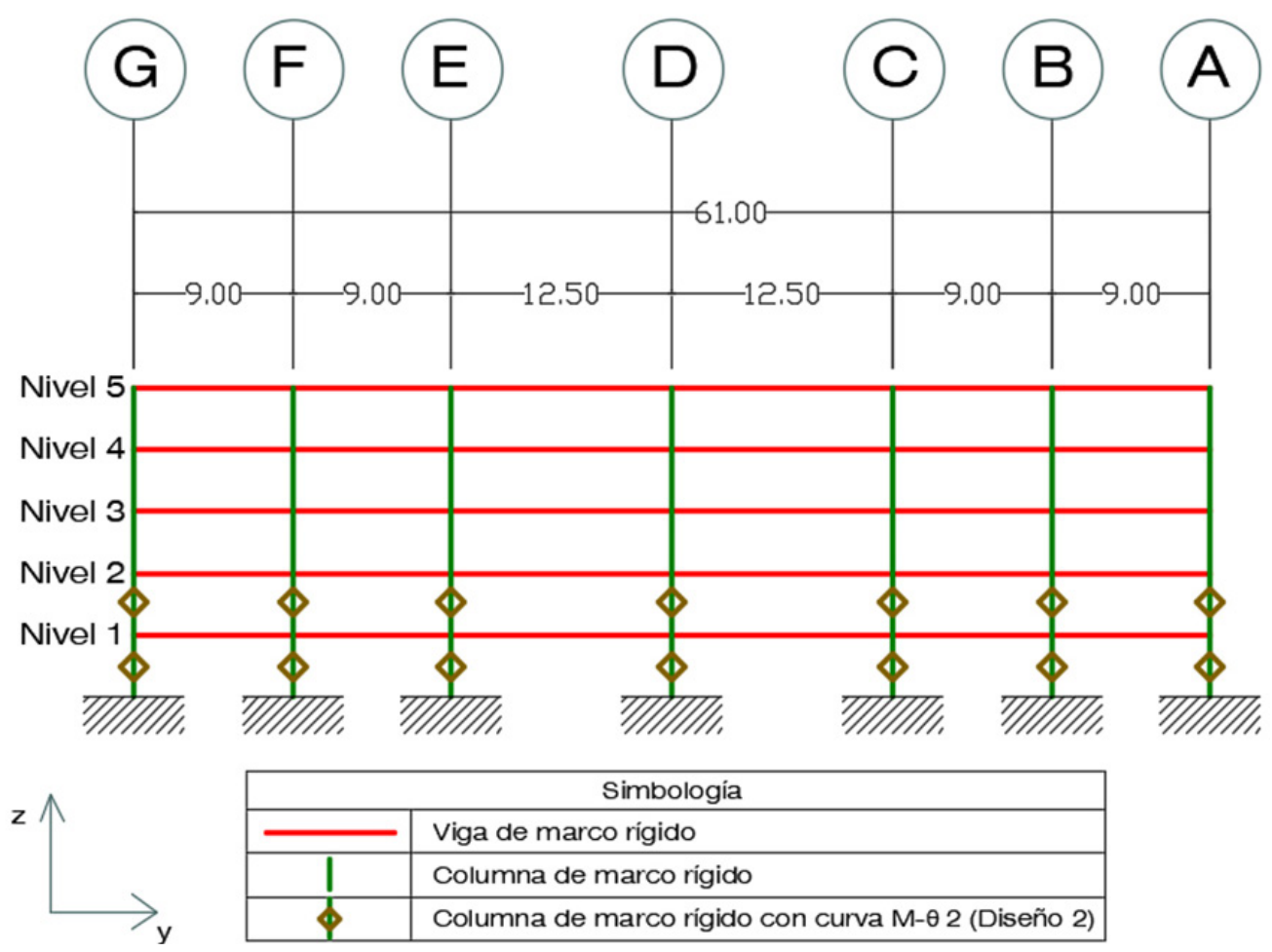

Figura 3. Elevación plano Y-Z (Unidades: Metros).

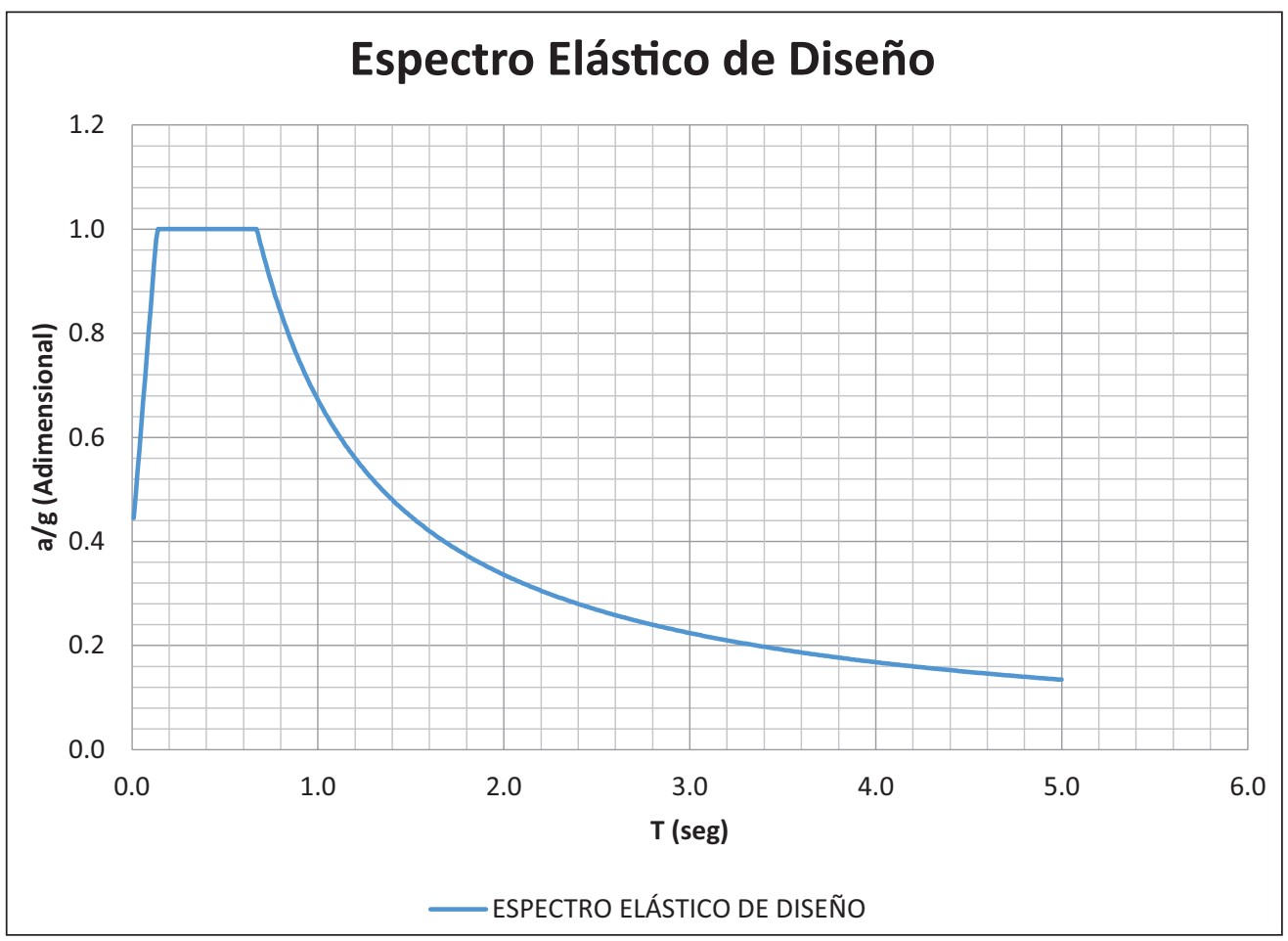

Figura 4. Espectro elástico de diseño.

Se evaluó el desempeño sísmico de los diseños realizando análisis no lineal estático y no lineal dinámico de tiempo-historia. Para cada tipo de análisis, las evaluaciones se realizaron tomando como base un grupo de objetivos de desempeño básico, el cual corresponde a estructuras ordinarias (15). El grupo de objetivos de desempeño se muestra en la Tabla 1.

La evaluación de cada diseño empleando análisis no lineal estático involucró la determinación de la demanda y capacidad de desplazamiento lateral de los mismos. En cada diseño y para cada nivel de desempeño la demanda se calculó por medio del método mejorado del espectro de capacidad, indicado por el FEMA 440. En dicho método se intercepta gráficamente el espectro de demanda sísmica de la estructura (reducido por parámetros que consideran la rigidez y amortiguamiento de la estructura dentro de su rango elástico, así como el comportamiento no lineal de la estructura) y el espectro de capacidad, obtenido de la curva de capacidad de la estructura. El 
Tabla 1. Objetivos de Desempeño Para Estructuras Ordinarias

\begin{tabular}{|c|c|c|}
\hline Movimiento de suelo & $\begin{array}{c}\text { Probabilidad de excedencia } \\
\text { en 5o años }\end{array}$ & Nivel de desempeño \\
\hline $\begin{array}{c}\text { Sismo frecuente } \\
(50 \% \text { del sismo de diseño) }\end{array}$ & $50 \%$ & Ocupación inmediata (IO) \\
\hline $\begin{array}{c}\text { Sismo de diseño } \\
\text { (sismo de acuerdo al UBC } 97)\end{array}$ & $10 \%$ & Seguridad de vida (LS) \\
\hline $\begin{array}{c}\text { Sismo máximo considerado } \\
(150 \% \text { del sismo de diseño) }\end{array}$ & $2 \%$ & Prevención al colapso (CP) \\
\hline
\end{tabular}

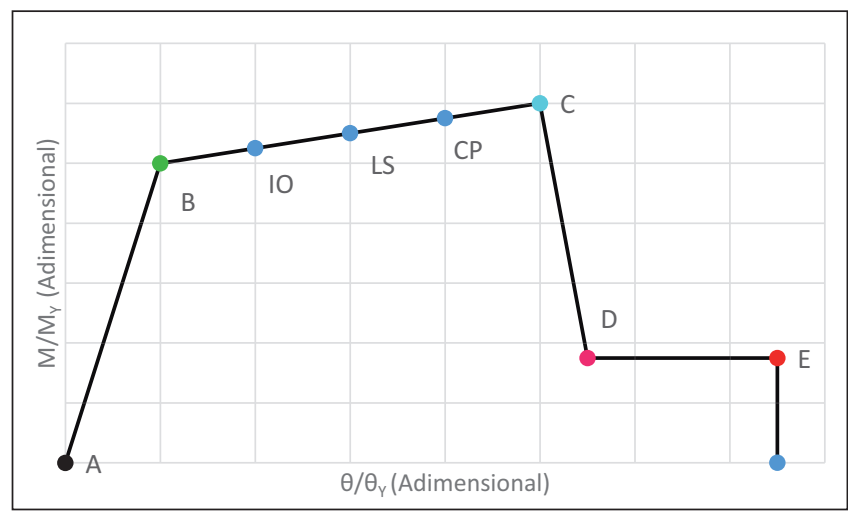

Figura 5. Curva momento - rotación en secciones transversales críticas de marcos rígidos.

punto de intercepción de ambas gráficas, o punto de desempeño, indica la demanda de desplazamiento lateral. La capacidad de desplazamiento lateral se calculó por medio de la curva de capacidad resultante del análisis no lineal realizado para cada diseño. La variación de fuerzas y deformaciones internas en las secciones transversales críticas de los marcos rígidos se estableció de acuerdo a la curva momento-rotación mostrada en la Figura 5. Las coordenadas de los puntos de dicha curva se determinaron de acuerdo a la Tabla 5-6 del FEMA 356.

Es importante mencionar que esta curva incluye los puntos que representan los criterios de aceptación del cumplimiento de los niveles de desempeño presentados en las secciones transversales críticas. De acuerdo al FEMA 356, la determinación de las coordenadas de los puntos de la curva se realiza considerando la relación ancho-espesor del alma y de los patines de los elementos estructurales de los marcos. También se considera la relación entre la resistencia a compresión axial requerida y la resistencia a compresión axial disponible de las secciones transversales en cuestión. En ese sentido se establecieron 2 curvas momento-rotación. Las coordenadas de los puntos que definen la curva 1 se muestran en las Tablas 2 y 3, mientras que las coordenadas de los puntos que definen la curva 2 se muestran en las Tablas 4 y 5 . La curva 1 fue asignada a las secciones transversales críticas de los marcos rígidos de todos los diseños, con la excepción de las secciones transversales críticas de las columnas indicadas en las Figuras 2 y 3, las cuales corresponden al diseño 2. A estas secciones transversales se les asignó la curva 2.

La evaluación del desempeño sísmico empleando análisis no lineal dinámico de tiempo-historia se realizó mediante la aplicación de tres historiales de aceleración de suelo sintéticos (acelerogramas sintéticos) sobre los marcos rígidos de cada diseño; tres por cada nivel de desempeño. Dichos acelerogramas sintéticos fueron creados siguiendo el método de empare-
Tabla 2. Coordenadas de los puntos de la curva momento rotación 1

\begin{tabular}{|l|c|c|}
\hline Punto/Coordenada & $\boldsymbol{\theta} / \boldsymbol{\theta}_{\mathbf{y}}$ & $\mathbf{M} / \mathbf{M}_{\mathbf{y}}$ \\
\hline A & 0 & 0 \\
\hline B & 0 & 1 \\
\hline C & 9 & 1.27 \\
\hline D & 9 & 0.6 \\
\hline E & 11 & 0.6 \\
\hline
\end{tabular}

Tabla 3. Criterios para los niveles de desempeño de la curva momento - rotación 1

\begin{tabular}{|l|c|}
\hline Nivel de desempeño & $\boldsymbol{\theta} / \boldsymbol{\theta}_{\mathbf{y}}$ \\
\hline Ocupación inmediata (IO) & $\mathbf{1}$ \\
\hline Seguridad de vida (LS) & 6 \\
\hline Prevención al colapso (CP) & 8 \\
\hline
\end{tabular}

Tabla 4. Coordenadas de los puntos de la curva momento rotación 2

\begin{tabular}{|l|c|c|}
\hline Punto/Coordenada & $\boldsymbol{\theta} / \boldsymbol{\theta}_{\mathbf{y}}$ & $\mathbf{M} / \mathbf{M}_{\mathbf{y}}$ \\
\hline A & 0 & 0 \\
\hline B & 0 & 1 \\
\hline C & 5.4 & 1.16 \\
\hline D & 5.4 & 0.2 \\
\hline E & 8.3 & 0.2 \\
\hline
\end{tabular}

Tabla 5. Criterios para los niveles de desempeño de la curva momento - rotación 2

\begin{tabular}{|l|c|}
\hline Nivel de desempeño & $\boldsymbol{\theta / \theta y}$ \\
\hline Ocupación inmediata (IO) & 0.25 \\
\hline Seguridad de vida (LS) & 3.92 \\
\hline Prevención al colapso (CP) & 5.39 \\
\hline
\end{tabular}

jamiento espectral en el dominio del tiempo (16) con base en tres registros de movimiento de suelo: Loma Prieta 17/10/89, estación Oakland Outer Harbor Wharf, componente $270^{\circ}$, Kobe 16/01/95, estación Kakogawa, componente $90^{\circ}$; y Northridge 17/01/94, estación Sylmar, CA, Converter Station East, componente $11^{\circ}$. Tales registros se representan en la Figura 6. 
Los acelerogramas sintéticos creados produjeron espectros de respuesta acordes con los espectros de demanda sísmica generados a partir del UBC 97 y que corresponden a cada uno de los movimientos de suelo indicados en la Tabla 1 (Sismos frecuente, de diseño y máximo considerado). En la Figura 7 se representan, para cada movimiento de suelo, los espectros de respuesta generados a partir de los acelerogramas sintéticos y los correspondientes espectros generados a partir del UBC

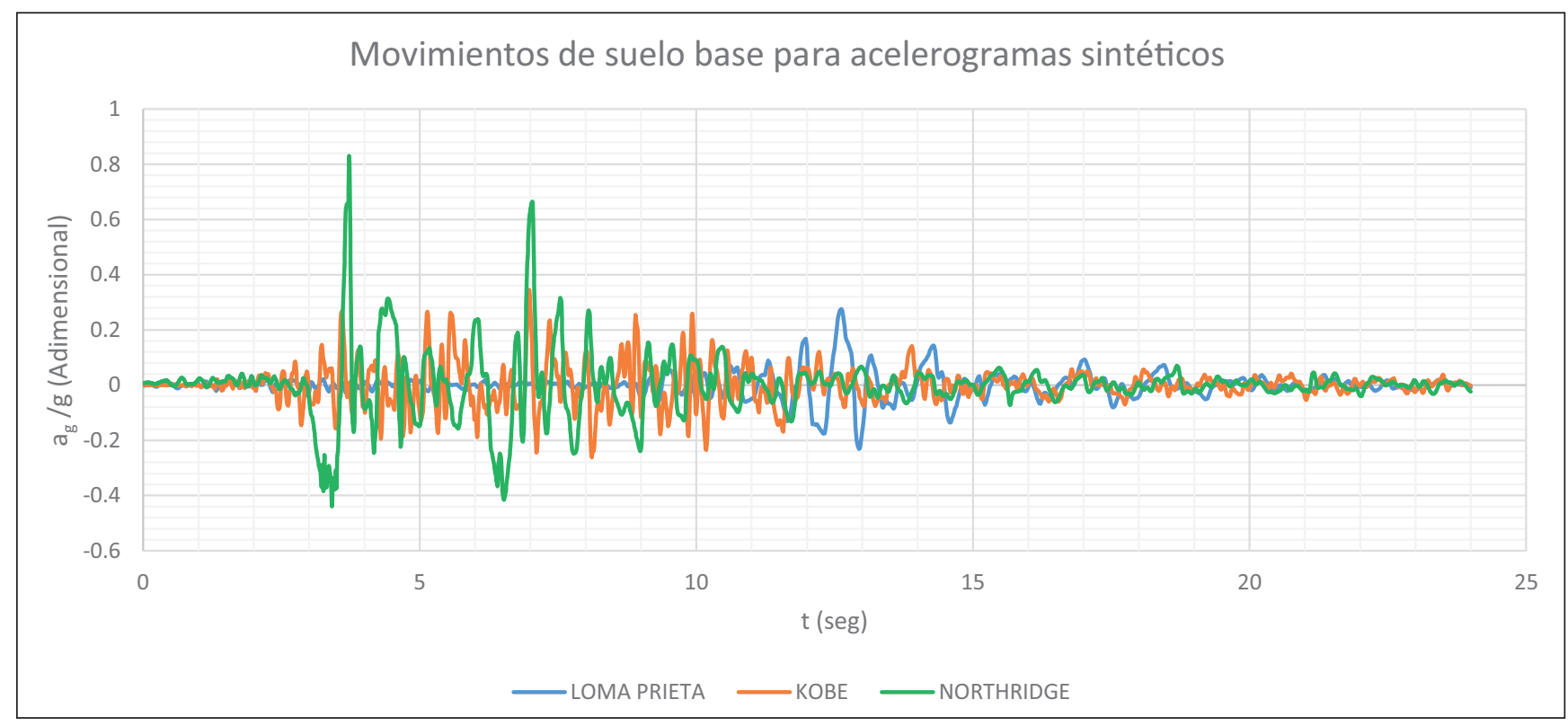

Figura 6. Movimientos de suelo base para la generación de acelerogramas sintéticos.

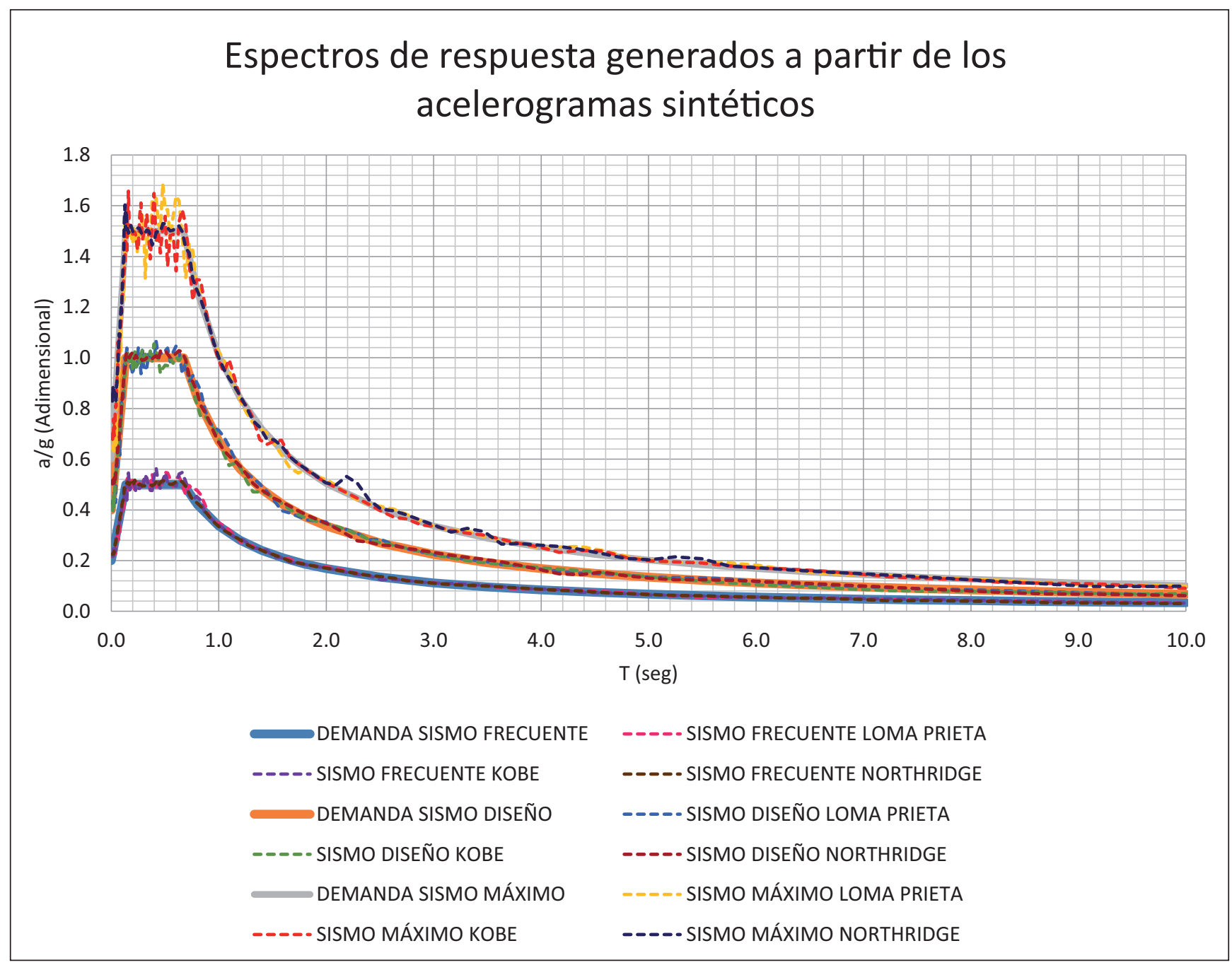

Figura 7. Espectros de respuesta generados a partir de los acelerogramas sintéticos 
97. Analizando los valores de aceleración correspondientes a los períodos pertinentes de cada diseño, se observó una diferencia porcentual inferior máxima de $1.82 \%$ y una diferencia porcentual inferior promedio de $0.80 \%$ entre los espectros de acelerogramas sintéticos y los espectros del UBC 97. Para efectos de los resultados de este trabajo, tales diferencias se consideraron despreciables.

Se determinó la respuesta de los diseños sometidos a los acelerogramas sintéticos mediante análisis no lineales dinámicos de tiempo-historia considerando el método de integración directa de Hilber, Hughes y Taylor (17). Se enfatiza que los modelos de los marcos rígidos empleados en los análisis no lineales dinámicos son los mismos que los modelos empleados en los análisis no lineales estáticos.

\section{ANÁLISIS DE RESULTADOS}

En las Tablas 6, 7 y 8 se muestran los perfiles seleccionados para los diseños de marcos rígidos 1, 2 y 3, respectivamente. $\mathrm{Al}$ emplear las secciones mostradas los marcos rígidos cumplieron con la resistencia requerida según el ANSI/AISC 34110 y con la rigidez lateral requerida establecida por el UBC 97.

\section{ANÁLISIS NO LINEALES ESTÁTICOS}

En las Figuras 8 a 13 se muestran las curvas de capacidad de los diseños 1, 2 y 3 en las direcciones X y Y. En dichas figuras se representa la demanda y capacidad de desplazamiento lateral de los marcos rígidos para los niveles de desempeño considerados en este proyecto (IO, LS, CP).

En las Figuras 8 a 13 se verifica que los diseños 1 y 3 cumplen con todos los niveles de desempeño considerados. En
Tabla 6. Secciones transversales del diseño 1

\begin{tabular}{|c|c|c|}
\hline \multirow{2}{*}{ Dirección } & Vigas & Columnas \\
\cline { 2 - 3 } & Sección & Sección \\
\hline $\mathbf{X}$ & $\mathrm{W}_{30 X 124}$ & $\mathrm{~W}_{3} 6 \mathrm{X}_{3} 61$ \\
\hline $\mathbf{Y}$ & $\mathrm{W}_{30 X 173}$ & $\mathrm{~W}_{3} 6 \mathrm{X}_{48} 87$ \\
\hline
\end{tabular}

Tabla 7. Secciones transversales del diseño 2

\begin{tabular}{|c|c|c|}
\hline \multirow{2}{*}{ Dirección } & Vigas & Columnas \\
\cline { 2 - 3 } & Sección & Sección \\
\hline $\mathbf{X}$ & $\mathrm{W}_{40 X} 662$ & W40X264 \\
\hline $\mathbf{Y}$ & $\mathrm{W}_{40 \mathrm{O}} 662$ & W40X264 \\
\hline
\end{tabular}

Tabla 8. Secciones transversales del diseño 3

\begin{tabular}{|c|c|c|}
\hline \multirow{2}{*}{ Dirección } & Vigas & Columnas \\
\cline { 2 - 3 } & Sección & Sección \\
\hline $\mathbf{X}$ & $\mathrm{W}_{33 \mathrm{X} 141}$ & $\mathrm{~W}_{3} 6 \mathrm{X} 262$ \\
\hline $\mathbf{Y}$ & $\mathrm{W}_{33 \mathrm{X} 201}$ & $\mathrm{~W}_{3} 6 \mathrm{X}_{3} 61$ \\
\hline
\end{tabular}

consecuencia y a la luz del método de análisis implementado, tienen un desempeño sísmico aceptable. Los marcos rígidos del diseño 2 solamente cumplen con el nivel de desempeño Ocupación Inmediata (IO). Debido a ello, no tienen un desempeño sísmico aceptable. Tales diseños no son apropiados para las demandas sísmicas aplicadas.

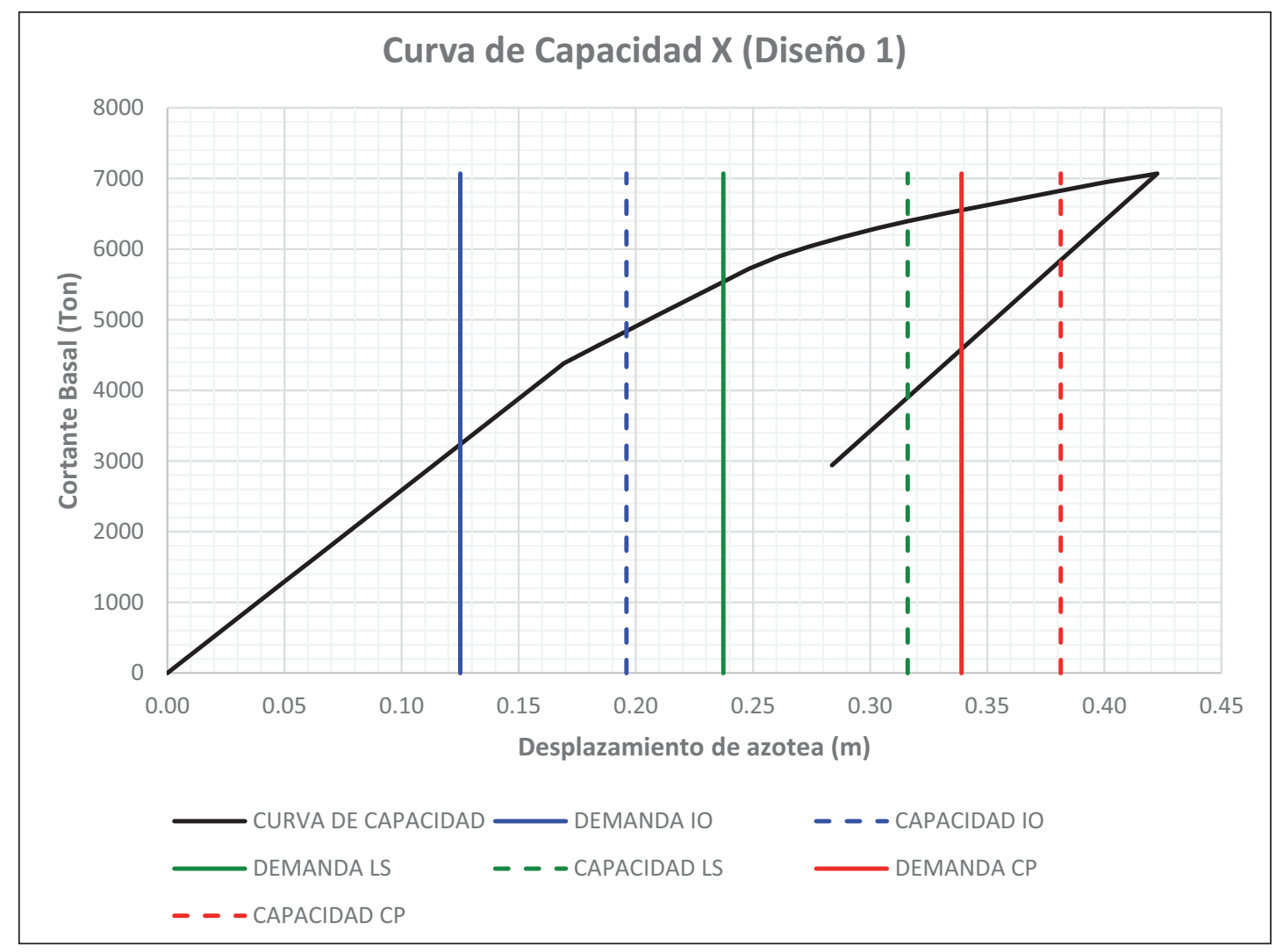

Figura 8. Curva de capacidad en X (Diseño 1). 


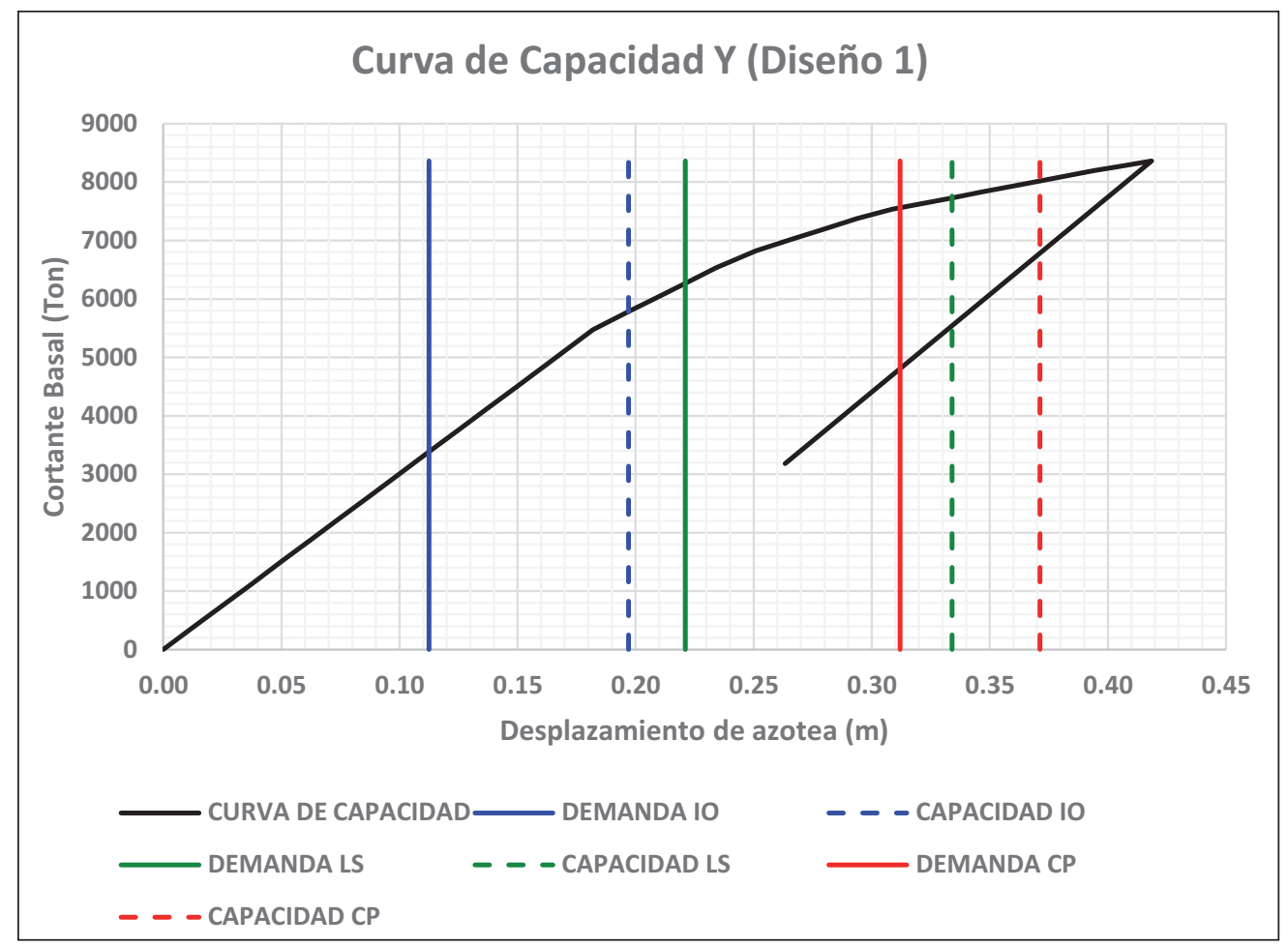

Figura 9. Curva de capacidad en Y (Diseño 1).

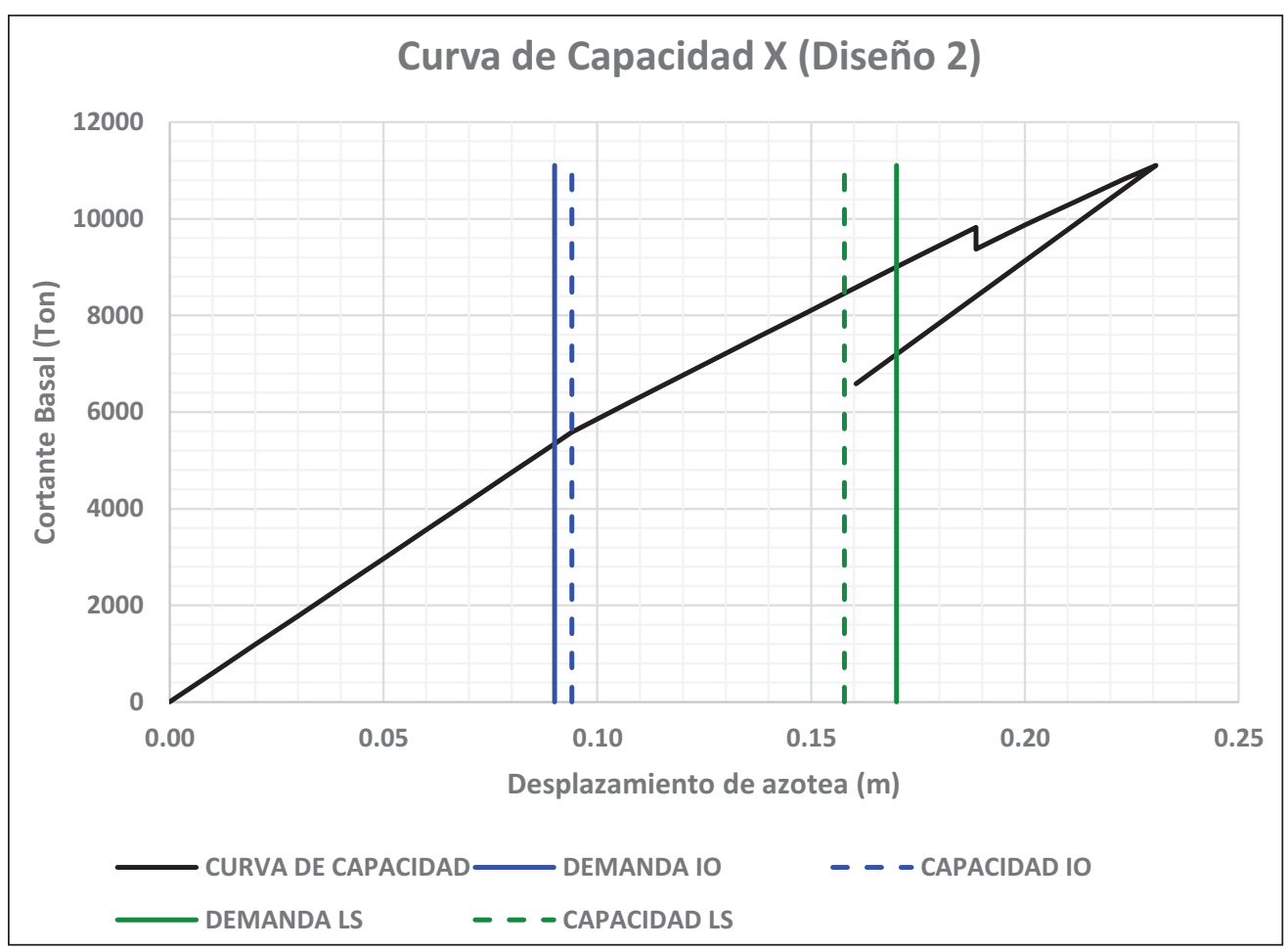

Figura 10. Curva de capacidad en X (Diseño 2).

En las Figuras 14 a 19 se muestran los mecanismos de falla presentados en los marcos debido a la aparición de articulaciones plásticas en las secciones transversales críticas de los elementos. En esas figuras aparece un código de colores que sirve para indicar la evolución de los momentos y rotaciones actuantes en las secciones transversales críticas a medida que se incrementa la fuerza lateral en la base. En la Tabla 9 se indica el color correspondiente a cada punto de la curva momento-rotación mostrada en la Figura 5.
Tabla 9. Código de colores del diagrama momento - rotación en secciones transversales críticas.

\begin{tabular}{|c|c|}
\hline Punto de la curva & Color \\
\hline B & Verde \\
\hline C & Celeste \\
\hline D & Magenta \\
\hline E & Rojo \\
\hline
\end{tabular}




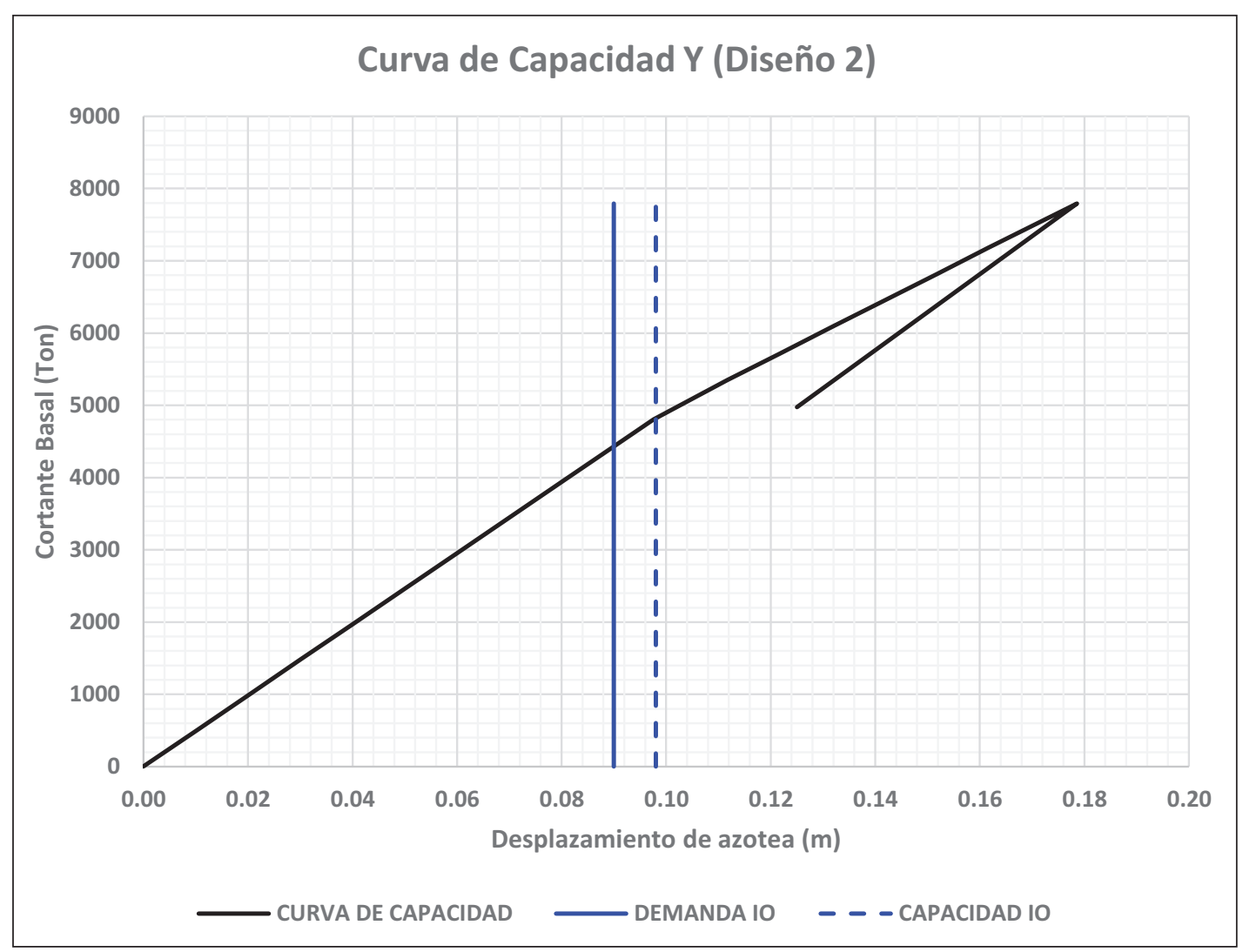

Figura 11. Curva de capacidad en Y (Diseño 2).

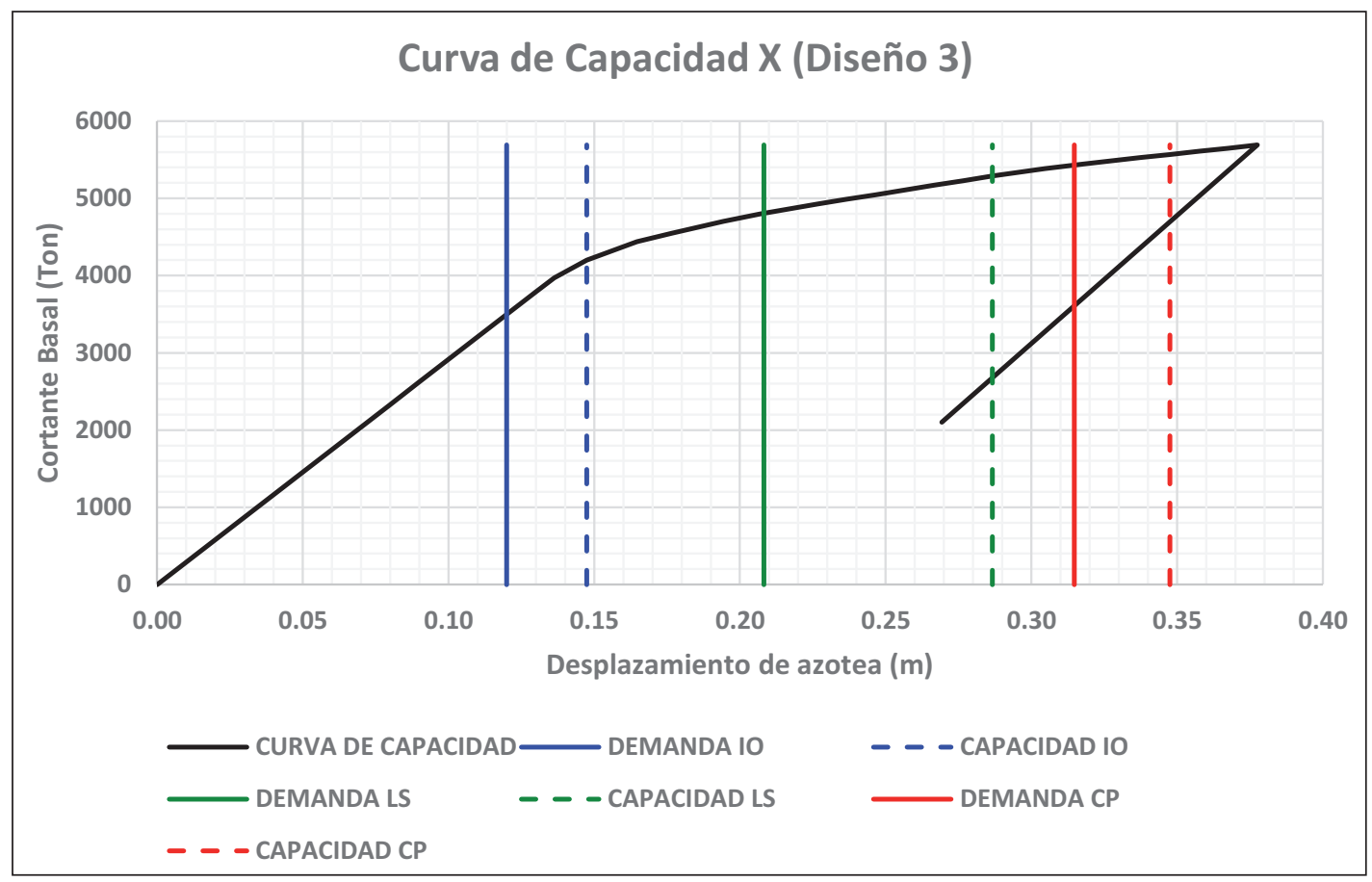

Figura 12. Curva de capacidad en X (Diseño 3).

De las Figuras 14 a 19 se nota que, a excepción de los marcos del diseño 2, todos los mecanismos de falla son deseables. Se caracterizan por la aparición de fluencias en las secciones críticas de las vigas antes que la formación de articulaciones plásticas en las secciones críticas de las columnas. Los mecanismos de falla de los marcos del diseño 2 son frágiles y, por consiguiente, indeseables. Tales mecanismos de falla se deben evitar.

\section{ANÁLISIS NO LINEALES DINÁMICOS}

En las Figuras 20 a 25 se presenta la respuesta final para los acelerogramas sintéticos de los sismos de Loma Prieta, Kobe y Northridge para los tres niveles de movimiento del suelo de la Tabla 1. De igual manera se indica el desplazamiento máximo absoluto (DMA) presentado por cada marco según el tipo de excitación aplicada. El código de colores mostra- 


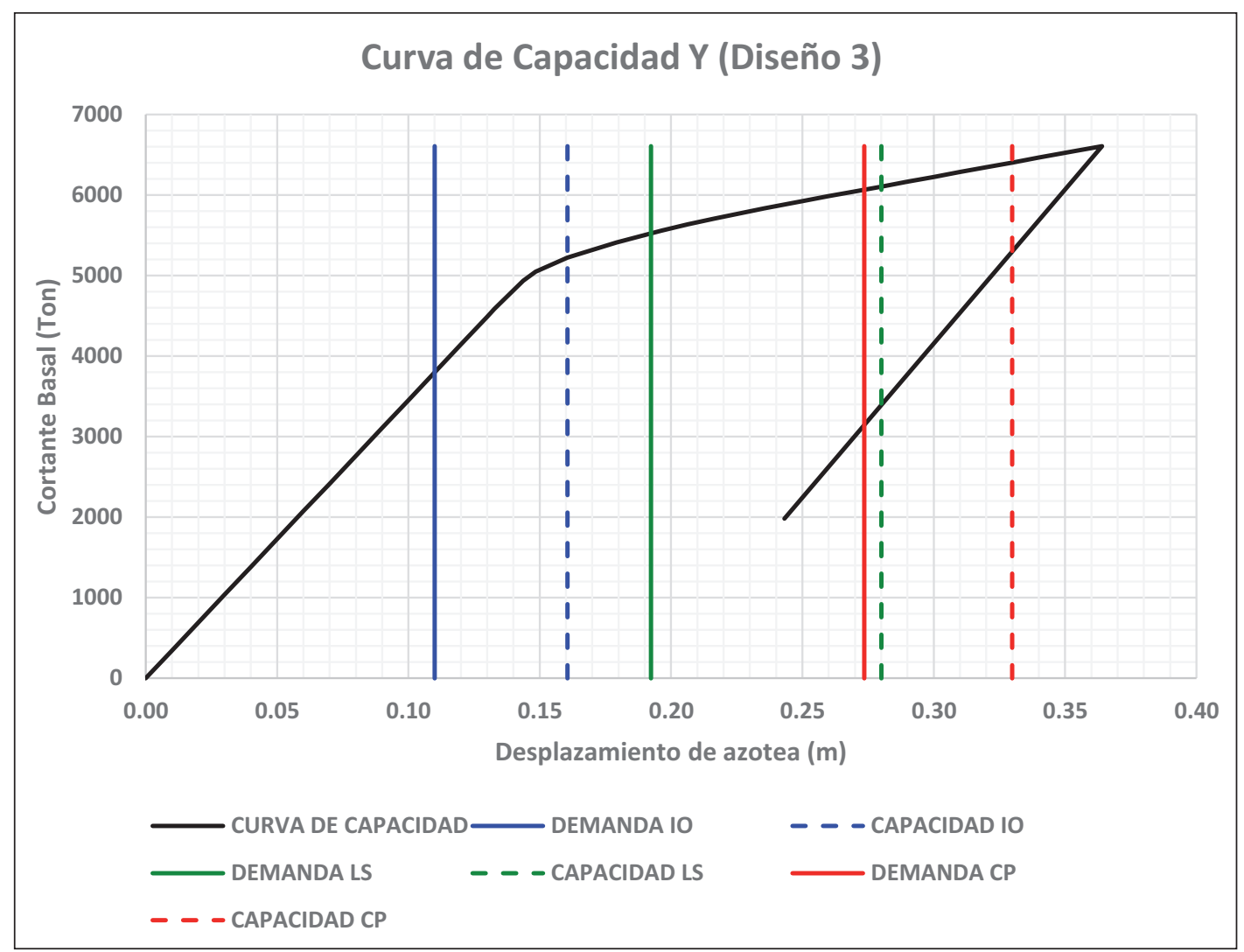

Figura 13. Curva de capacidad en Y (Diseño 3).

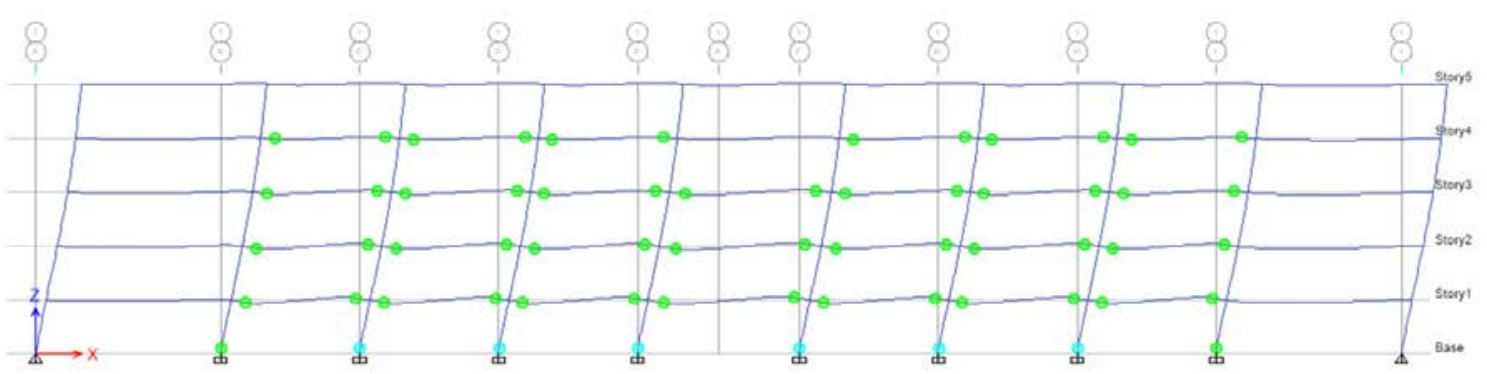

Figura 14. Mecanismo de falla en marcos en X del diseño 1 (desplazamiento de azotea: $0.423 \mathrm{~m}$ ).

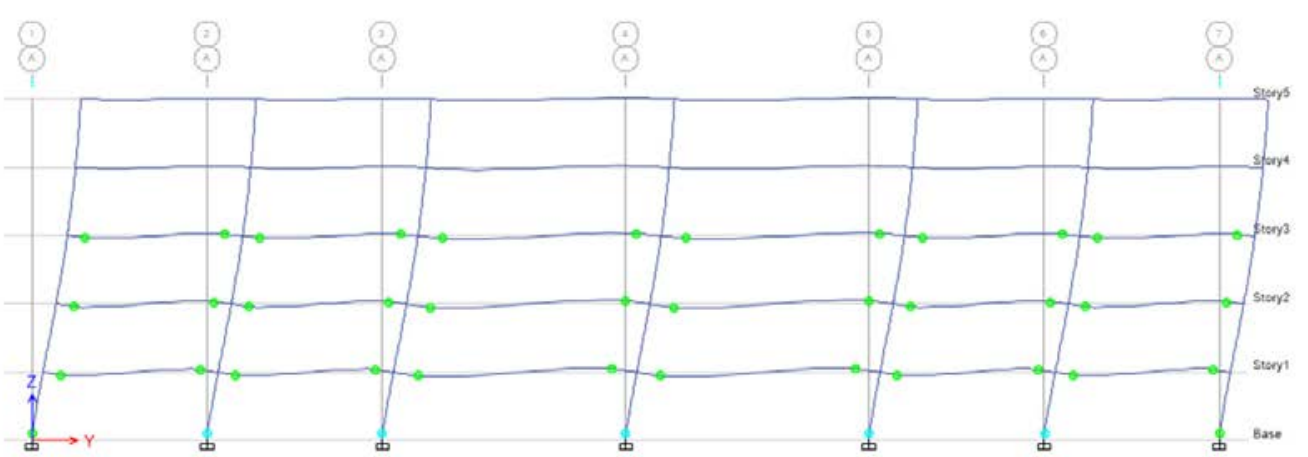

Figura 15. Mecanismo de falla en marcos en Y del diseño 1 (desplazamiento de azotea: 0.418 m). 


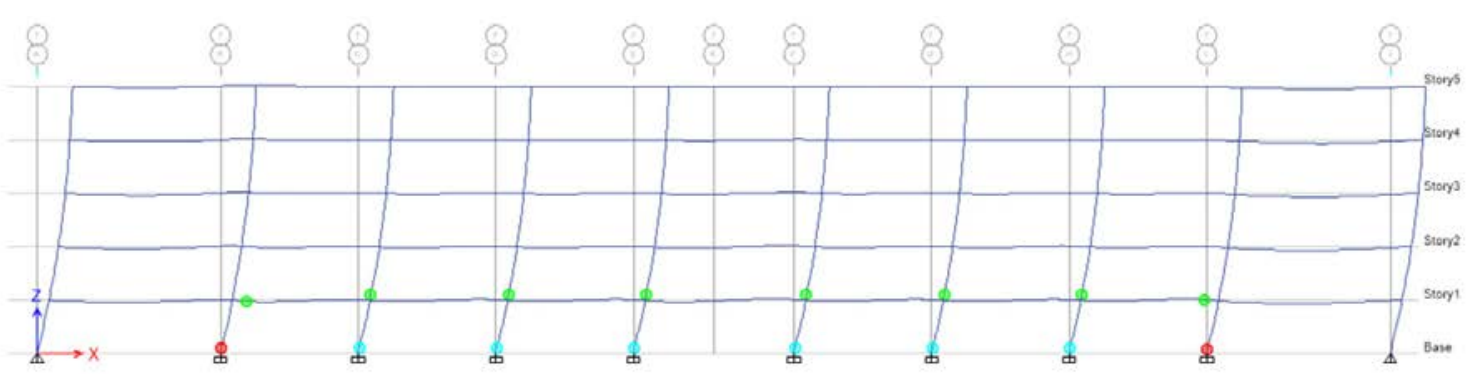

Figura 16. Mecanismo de falla en marcos en X del diseño 2 (desplazamiento de azotea: $0.231 \mathrm{~m}$ ).

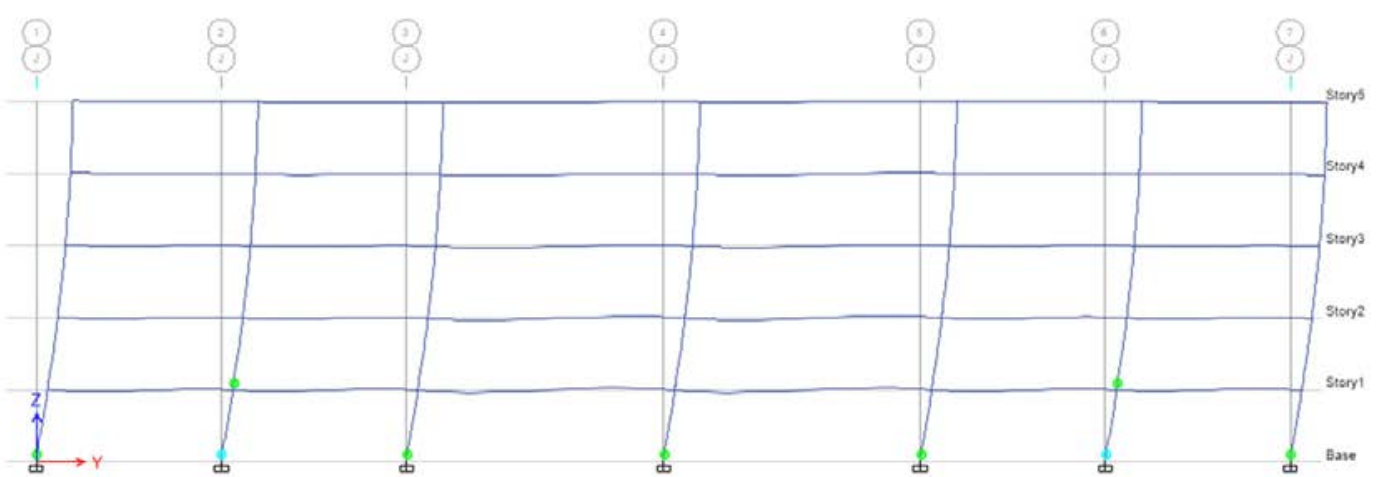

Figura 17. Mecanismo de falla en marcos en Y del diseño 2 (desplazamiento de azotea: $0.179 \mathrm{~m}$ ).

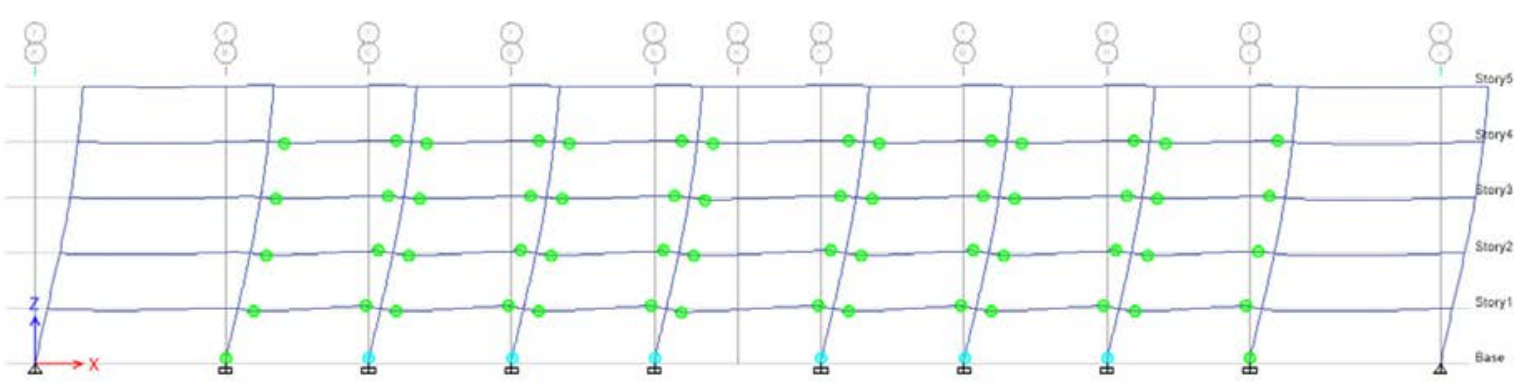

Figura 18. Mecanismo de falla en marcos en X del diseño 3 (desplazamiento de azotea: $0.378 \mathrm{~m}$ ).

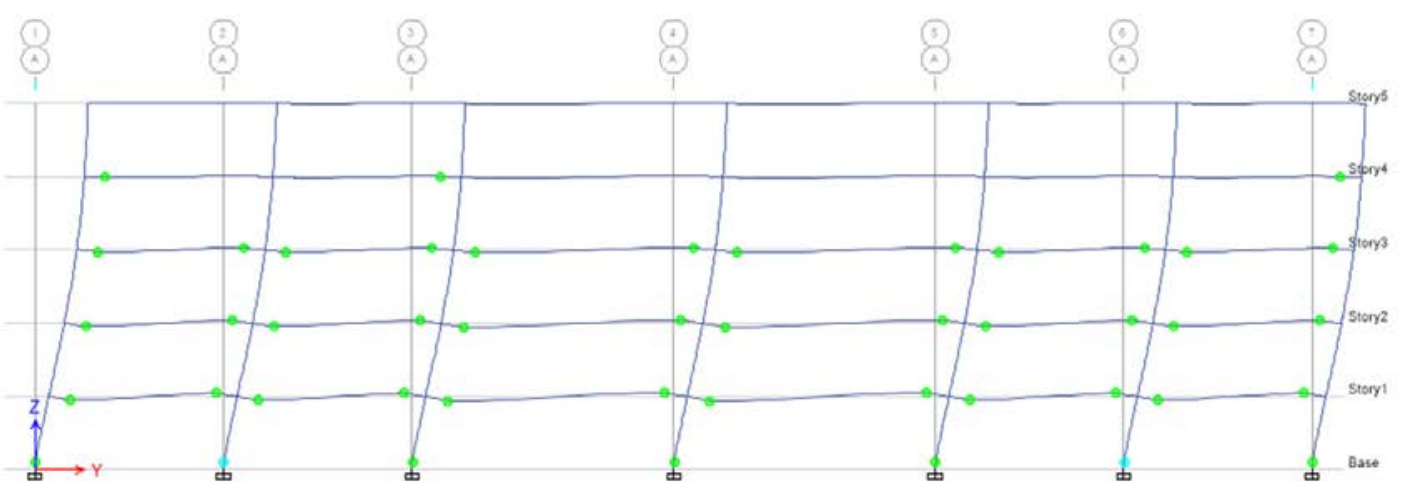

Figura 19. Mecanismo de falla en marcos en Y del diseño 3 (desplazamiento de azotea: 0.364 m). 
do en las figuras indica el nivel de desempeño presentado en las secciones transversales críticas de los elementos a través del tiempo. En la Tabla 10 se indica el color correspondiente a cada nivel de desempeño. Tal como sucede en el caso de análisis no lineales estáticos, los resultados muestran que los diseños 1 y 3 cumplen con los niveles de desempeño considerados dando como resultado un desempeño sísmico aceptable. En estos diseños las articulaciones plásticas ocurren los extremos de las vigas y en las bases de las columnas, sin producirse un mecanismo en las columnas. En contraste, los

\begin{tabular}{|c|c|c|c|}
\hline & SISMO FRECUENTE & SISMO DE DISEÑO & SISMO MÁXIMO CONSIDERADO \\
\hline $\begin{array}{l}\text { SINTÉTICO } \\
\text { LOMA PRIETA }\end{array}$ & 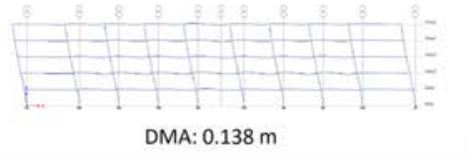 & 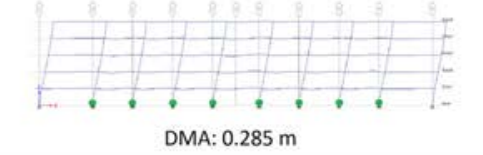 & 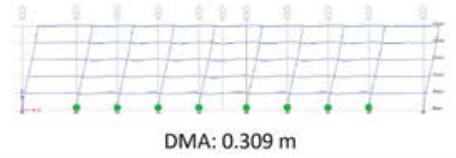 \\
\hline $\begin{array}{l}\text { SINTÉ́TICO } \\
\text { KOBE }\end{array}$ & 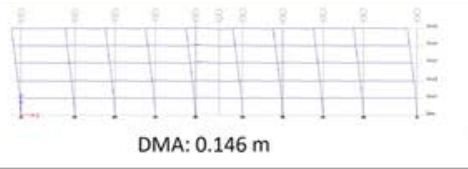 & 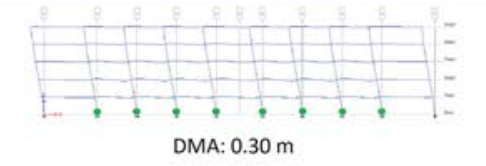 & 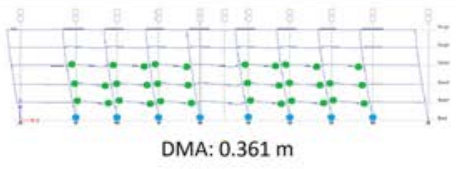 \\
\hline $\begin{array}{l}\text { SINTÉTICO } \\
\text { NORTHRIDGE }\end{array}$ & 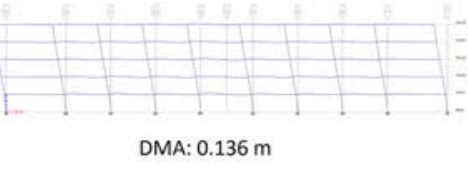 & 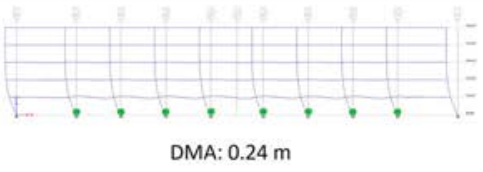 & $\because \because \because ; \quad \because \because \cdots ;$ \\
\hline & 10 & LS & $C P$ \\
\hline
\end{tabular}

Figura 20. Estado final de los marcos en $\mathrm{X}$ del diseño 1 al ser sometidos a los movimientos de suelo.

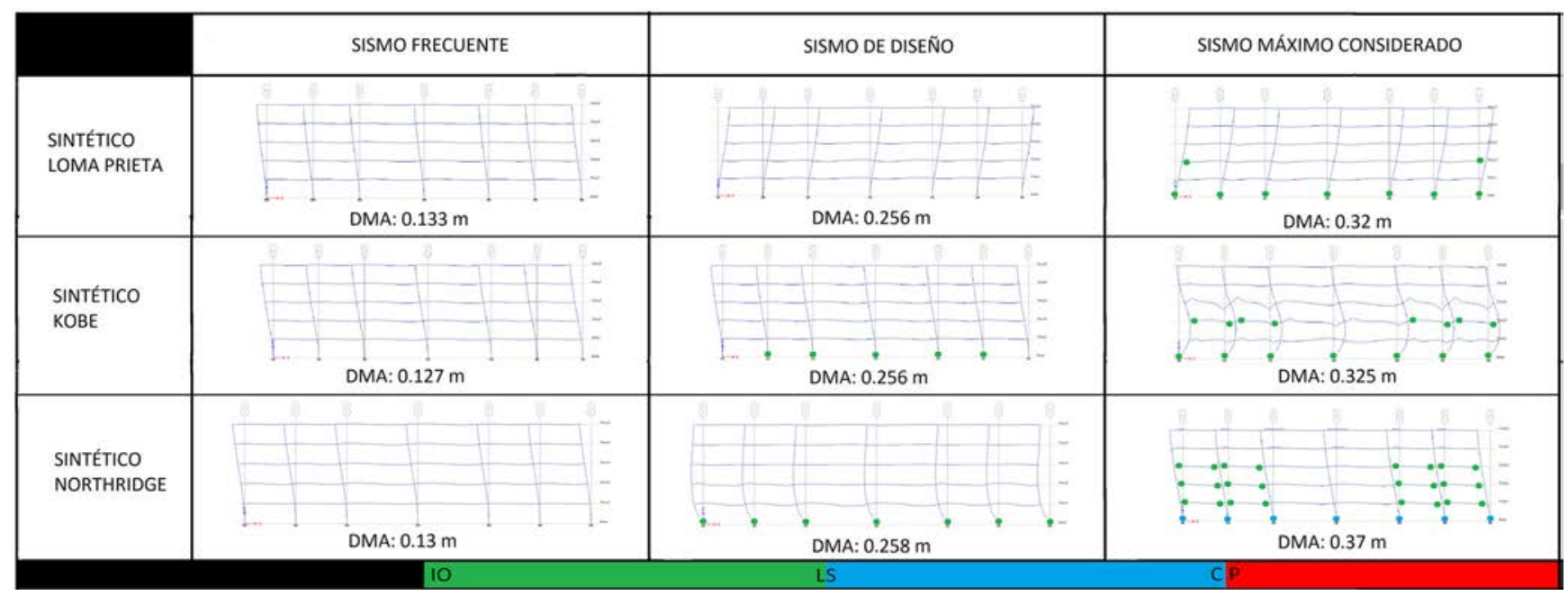

Figura 21. Estado final de los marcos en Y del diseño 1 al ser sometidos a los movimientos de suelo.

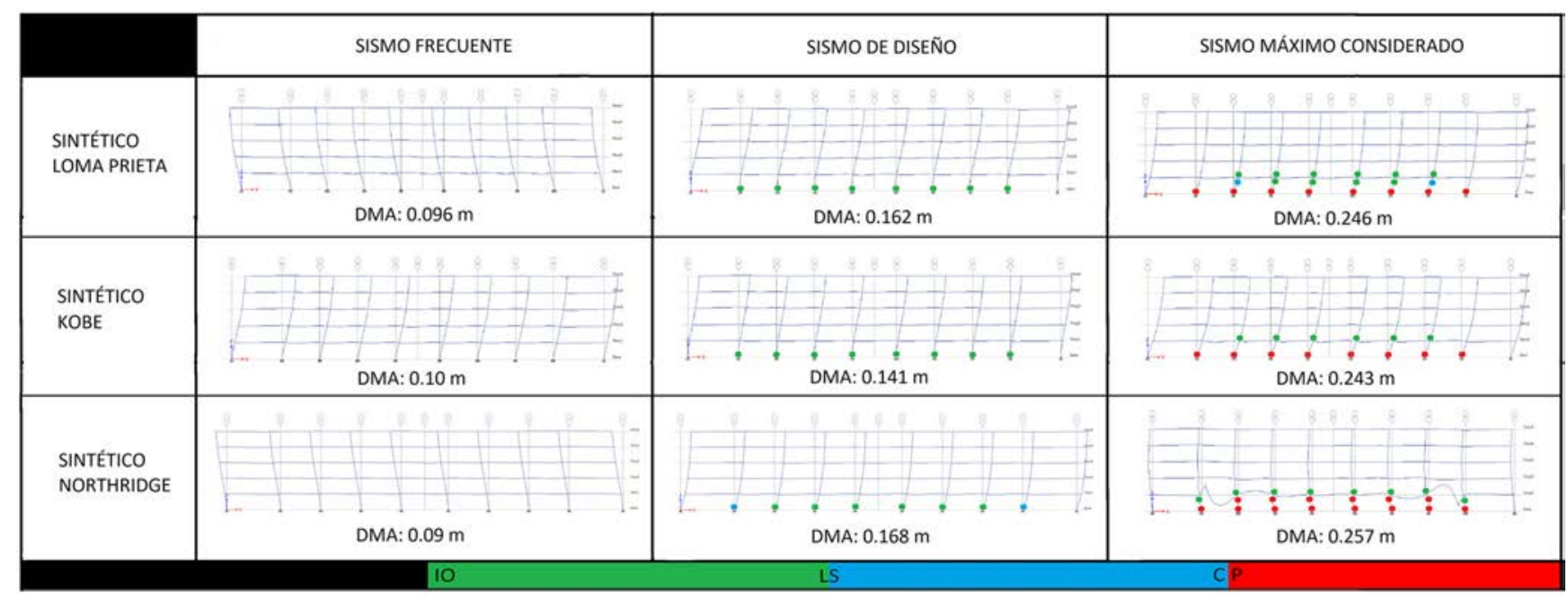

Figura 22. Estado final de los marcos en $\mathrm{X}$ del diseño 2 al ser sometidos a los movimientos de suelo. 
resultados del diseño 2 muestran articulaciones plásticas en los extremos de las columnas, generando así el mecanismo no deseado de piso suave en la dirección X para el acelerograma sintético de Northridge así como para los tres acelerogramas sintéticos en la dirección Y. Lo anterior para el máximo sismo considerado.

\section{CONCLUSIONES}

De acuerdo con los resultados obtenidos en el presente estudio, los diseños 1 y 3 cumplen con la normativa UBC $97 \mathrm{y}$ ANSI/AISC 2010 y presentan un desempeño sísmico aceptable.

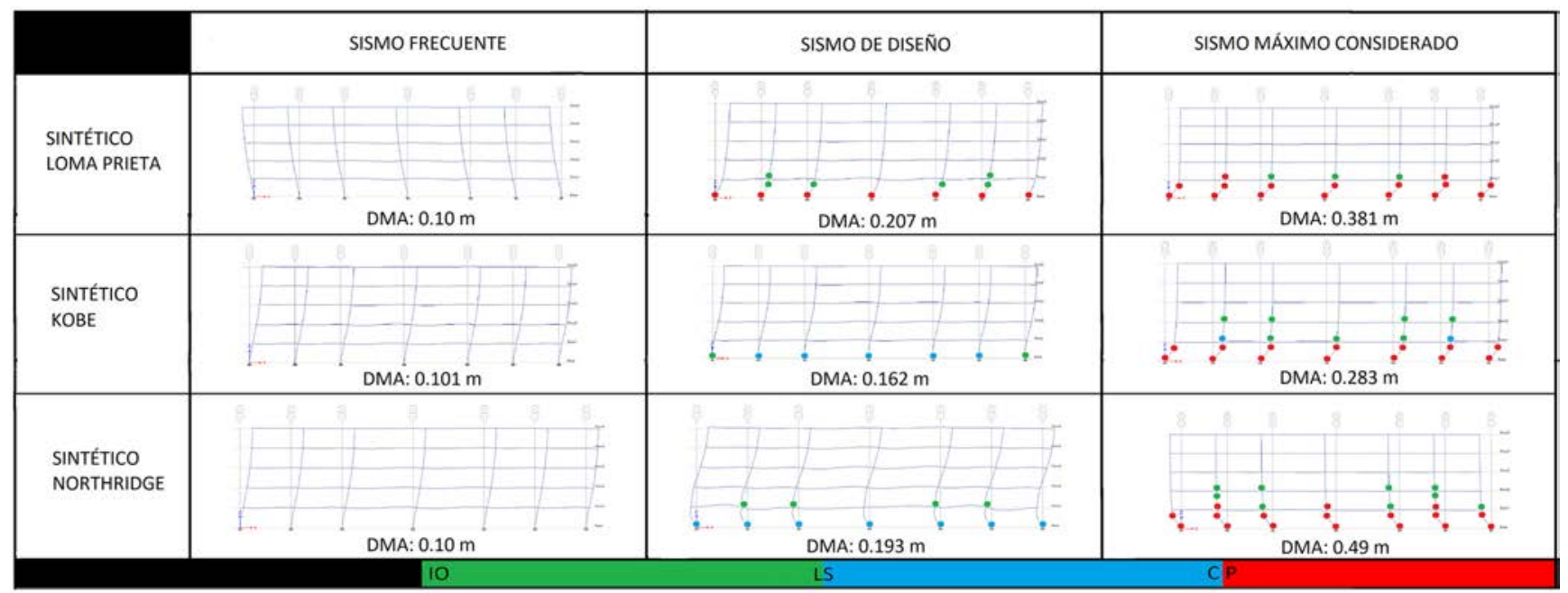

Figura 23. Estado final de los marcos en $Y$ del diseño 2 al ser sometidos a los movimientos de suelo.

\begin{tabular}{|c|c|c|c|c|}
\hline & & SISMO FRECUENTE & SISMO DE DISEÑO & SISMO MÁXIMO CONSIDERADO \\
\hline $\begin{array}{l}\text { SINTÉTICO } \\
\text { LOMA PRIETA }\end{array}$ & 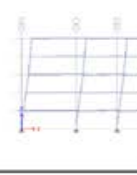 & $\lim _{\text {DMA: } 0.132 \mathrm{~m}}$ & $\because \because \because \because \because \because \because \because \because \vdots: \because \cdots$ & $\because \because \because \because \because \because \because \because \because \vdots:$ \\
\hline $\begin{array}{l}\text { SINTÉTICO } \\
\text { KOBE }\end{array}$ & 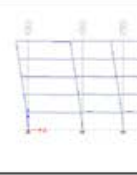 & $\lim _{\text {DMA: } 0.125 \mathrm{~m}}$ & $\vdots \because \because \because \vdots \vdots \because \because \because \vdots \vdots: \cdots$ & $\because \because \because \because \because \because \because \because \because: \because \because: \because \because:$ \\
\hline $\begin{array}{l}\text { SINTÉTICO } \\
\text { NORTHRIDGE }\end{array}$ & 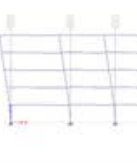 & DMA: $0.131 \mathrm{~m}$ & 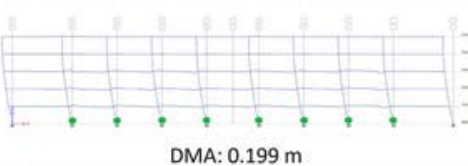 & $\vdots \because \because \because \vdots: \because \because \because \because \vdots:$ \\
\hline
\end{tabular}

Figura 24. Estado final de los marcos en X del diseño 3 al ser sometidos a los movimientos de suelo.

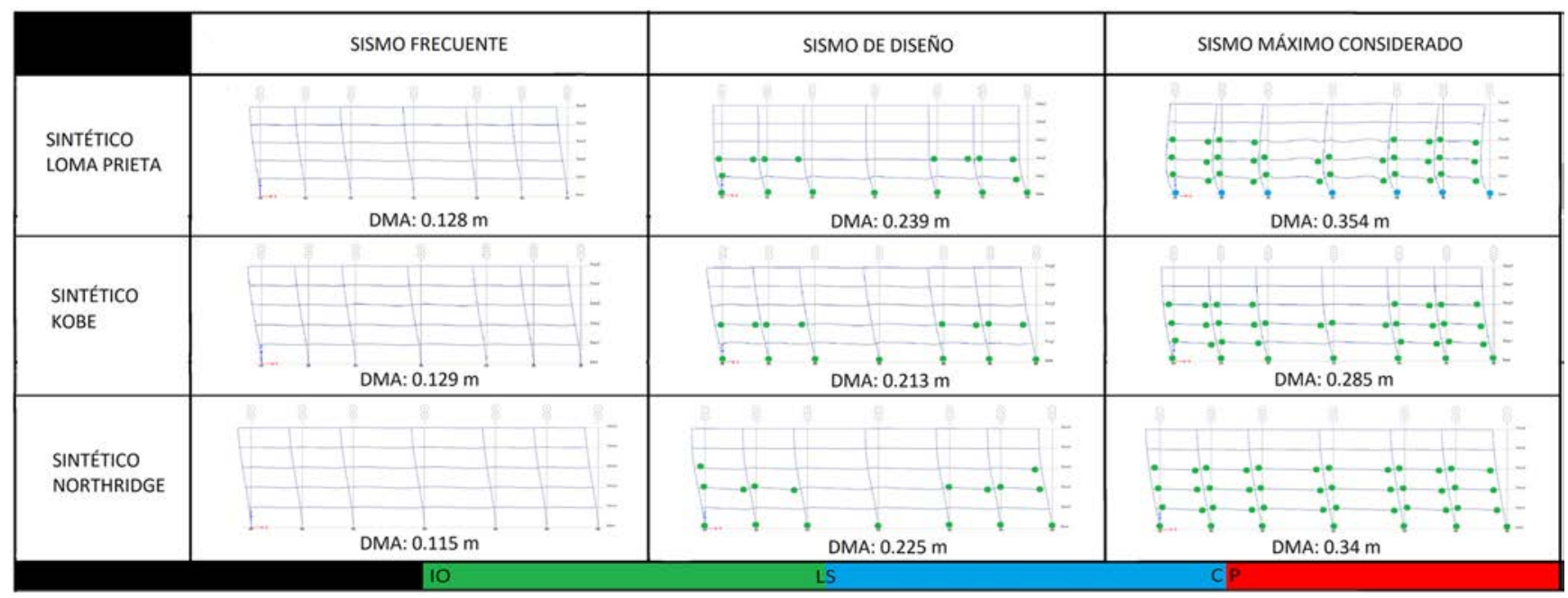

Figura 25. Estado final de los marcos en Y del diseño 3 al ser sometidos a los movimientos de suelo. 
Tabla 10. Código de colores de niveles de desempeño en secciones transversales críticas.

\begin{tabular}{|c|c|}
\hline Nivel de desempeño & Color \\
\hline Ocupación inmediata (IO) & Verde \\
\hline Seguridad de vida (LS) & Azul \\
\hline Prevención al colapso (CP) & Rojo \\
\hline
\end{tabular}

El comportamiento del diseño 2 indica que este tipo de diseño, a pesar de ser permitido por el CHOC y cumplir con la normativa UBC 97 y AISC 2010, tiene un desempeño sísmico inaceptable por lo que es inseguro e inapropiado para la demanda sísmica aplicada. Se deben incorporar restricciones en el CHOC al respecto.

La aportación de este artículo descansa en la identificación de una modificación necesaria en el CHOC que, de no hacerse, podría conducir a diseños inaceptables o, incluso, fallas con grandes pérdidas económicas y pérdida de vidas humanas.

\section{RECOMENDACIONES}

Se recomienda investigar el grado de propensión que existe hacia la generación de un diseño frágil, tal y como el que se presenta en este trabajo, de tal forma que dicha investigación pueda servir para la emisión de una restricción normativa confiable.

\section{AGRADECIMIENTOS}

Se agradece la colaboración económica brindada por la Dirección de Investigación de la Universidad Tecnológica Centroamericana para esta investigación. Se agradecen las aportaciones brindadas por Carlos Reyes ${ }^{(+)}$, Mario Covarrubias y los alumnos de la Universidad Tecnológica Centroamericana Selvin Euraque y Miguel Lanza.

\section{REFERENCIAS}

(1) Zameeruddin, M., Sangle, K. (2016). Review on Recent developments in the performance-based seismic design of reinforced concrete structures. Structures, 6: 119-133. https://doi.org/10.1016/j.istruc.2016.03.001

(2) Bertero, R., Bertero, V. (2002). Performance-based seismic engineering: the need for a reliable conceptual comprehensive approach. Earthquake engineering and structural dynamics, 31(3): 627-652. https://doi.org/10.1002/eqe.146

(3) NCEER. (1994). The Northridge, California Earthquake of January 17, 1994: General Reconnaissance Report, p. 3-28, Buffalo: National Center for Earthquake Engineering Research.

(4) NCEER. (1995). NCEER Response, p.10, Buffalo: National Center for Earthquake Engineering Research.

(5) CICH. (2010). Código Hondureño de Construcción: Normas Técnicas. Colegio de Ingenieros Civiles de Honduras.

(6) ICBO. (1994). Uniform Building Code. International Conference of Building Officials.

(7) Liu, B.-q., Liu, M., \& Li, Y.-b. (2004). Research and Development of Performance-Based Seismic Design Theory. Recuperado de http://www.iitk.ac.in/nicee/wcee/article/13_2457.pdf

(8) Naeim, F. (2001). The Seismic Design Handbook, p. 129, Norwell: International Code Council-Structural Engineers Association National Council. https://doi.org/10.1007/978-1-4615-1693-4

(9) ICBO. (1997). Uniform Building Code. International Conference of Building Officials.

(10) AISC. (2010). Specification for Structural Steel Buildings. American Institute of Steel Construction.

(11) AISC. (2010). Seismic Provisions for Structural Steel Buildings. American Institute of Steel Construction.

(12) FEMA. (2005). Improvement of Nonlinear Static Seismic Analysis Procedures. Federal Emergency Management Agency.

(13) FEMA. (2000). Prestandard and Commentary for the Seismic Rehabilitation of Buildings. Federal Emergency Management Agency.

(14) AISC. (2011). Steel Construction Manual. American Institute of Steel Construction.

(15) FEMA. (2009). NEHRP Recommended Seismic Provisions for New Buildings and Other Structures. Federal Emergency Management Agency.

(16) Al Atik, L., \& Abrahamson, N. (2010). An Improved Method for Nonstationary Spectral Matching. Earthquake Spectra, 26(3): 601-617. https://doi.org/10.1193/1.3459159

(17) Hilber, H., Hughes, T., \& Taylor, R. (1977). Improved Numerical Dissipation For Time Integration Algorithms in Structural Dynamics. Earthquake Engineering And Structural Dynamics, 5(3): 283-292. https://doi.org/10.1002/ eqe.4290050306 